\title{
Visual discrimination learning in the killer whale (Orcinus orca)
}

\author{
DON WHITE, PAUL SPONG, NORM CAMERON, and JOHN BRADFORD \\ Cetacean Research Laboratory, Division of Neurological Sciences \\ University of British Columbia, Vancouver 8, B.C., Canada
}

Trial-and-error and errorless training procedures were used to train two killer whales on a simple visual discrimination problem. Both whales demonstrated difficulties in solving the problem under trial-and-error training. The relative effectiveness of the two training procedures is evaluated.

Difficulties in training cetacea for visual experiments have been reported by several investigators. Kellogg and Rice (1966), investigating problem solving in a bottle-nosed dolphin, began pattern discrimination using trial-and-error training. After "hundreds of trials" the dolphin failed to solve the task, and errorless training (successive approximations) was used to bring the animal to a criterion level of performance. Spong and White (1971), measuring the visual acuity of the Pacific white-sided dolphin, found no evidence that the dolphin had learned to discriminate between a white card with two black lines and a blank white card after 600 trials.

The present report summarizes the performance of two killer whales on a visual discrimination task which constituted preliminary training for later experiments in stimulus generalization and visual acuity. Both whales demonstrated difficulties in solving a simple visual discrimination under trial-and-error training, as did the dolphin in the Kellog and Rice and the Spong and White studies noted above. Accordingly, the present report is oriented towards an evaluation of methods of training used in visual discrimination experiments with cetacea.

\section{Subjects}

The Ss were two killer whales (Orcinus orca) at the Vancouver Public Aquarium. A female (Skana), approximately 6 years old, was housed in an exhibition pool open to the public. The second whale, a 3-year-old male (Hyak), was kept in an isolated research pool.

\section{Apparatus}

The two-choice visual discrimination apparatus consisted of two $12 \times 24$ in. clear Plexiglas windows set into a $42 \times 60$ in. sheet of plywood. Levers (6 616 in.), extending horizontally above each window, could be raised through 45-deg closing microswitches housed apparatus was submerged to the top of the windows, the levers were approximately $4 \mathrm{in}$. above water level. A doorbell was used to signal trial onset and a floodlight to signal correct responses. Slots for displaying stimulus cards were located immediately behind the Plexiglas windows. All responses were automatically recorded on an event recorder.

In the initial experimental session, each killer whale was shaped, using successive approximations, to raise either lever to the apparatus bumper block. Two additional sessions were devoted to training the whales to wait in a starting position approximately $15 \mathrm{ft}$ in front of the apparatus (by withholding trial onset) and to make nonpreferential responses to the two levers (reward was discontinued after more than four consecutive responses to the same lever). Discrimination training began in the fourth session.

The CS+ was a white Plexiglas card, $12 \times 26$ in., with two vertical black lines, each line $1 \mathrm{in}$. wide and $12 \mathrm{in.}$ long. A gap of $2^{1 / 2}$ in. was centered on the card. Negative stimuli were a blank white Plexiglas card and a blank black Plexiglas card (same dimensions). When the whale was in the starting position, the bell was rung for 1 sec, signaling the start of a trial, and the stimulus cards were dropped into the slots immediately behind the display windows. If the response was correct (i.e., the switch above the two-line card was closed), the floodlight on the apparatus was automatically turned on for $3 \mathrm{sec}$, two herring were dropped into the pool in front of the apparatus, and the stimulus cards were removed. The next trial began when the whale returned to the starting position. If the response was incorrect, a time-out period of 1 min was interposed before the start of the next trial.

Presentation of the CS+ in each of the two display windows and the appearance of each negative stimulus was randomized, with the constraint of equal exposure for each combination in a block of 24 trials. within bumper blocks. When the
Training sessions consisted of $\mathbf{7 2}$ discrete trials, with criterion for solution of the problem set at $90 \%$ correct over a 72 -trial session. If an animal failed to solve the discrimination problem after 2,000 trials, use of the negative stimuli was temporarily discontinued and the whale was conditioned to respond consistently to the CS+. The negative stimuli were then gradually reintroduced.

\section{RESULTS AND DISCUSSION}

Visual trial-and-error learning appears to be difficult for the killer whale as well as for the dolphin. Skana's trials to criterion for the problem totalled $1,656,828$ presentations of the black CS- and 828 presentations of the white CS-. Hyak failed to discriminate between the CS+ and the white CS- within 1,980 presentations using this procedure.

Figure 1A displays Skana's percent correct responses to each negative stimulus during trial-and-error training; Fig. 1B presents similar data for Hyak. There is no indication of differential responding to negative stimuli in Fig. 1A. Hyak, however, learned to discriminate between the $\mathrm{CS}+$ and the black CS- early in training (Fig. 1B), the use of this negative stimulus then being discontinued. Responses on trials presenting the black CS- were $97 \%$ correct after 288 trials, and had not fallen below $95 \%$ correct when the CS- was eliminated. Correct responses to the white CS- remained at chance levels throughout trial and error training.

It is not immediately obvious why one whale attended to the more apparent of the brightness discriminations (CS+ vs black CS-) and the second did not (Fig, 1). Apparently, different aspects of the stimuli acquired control over responding in the two Ss. The data thus suggest that contrast alone is an inefficient base on which to conduct either trial-and-error learning or errorless training.

Position preferences were formed immediately by both animals and were remarkably hard to discourage. With Skana, a period of correction procedure (repeat trials) was introduced after 300 trials and remained in effect until 936 trials had been completed. A period of correction procedure was introduced 


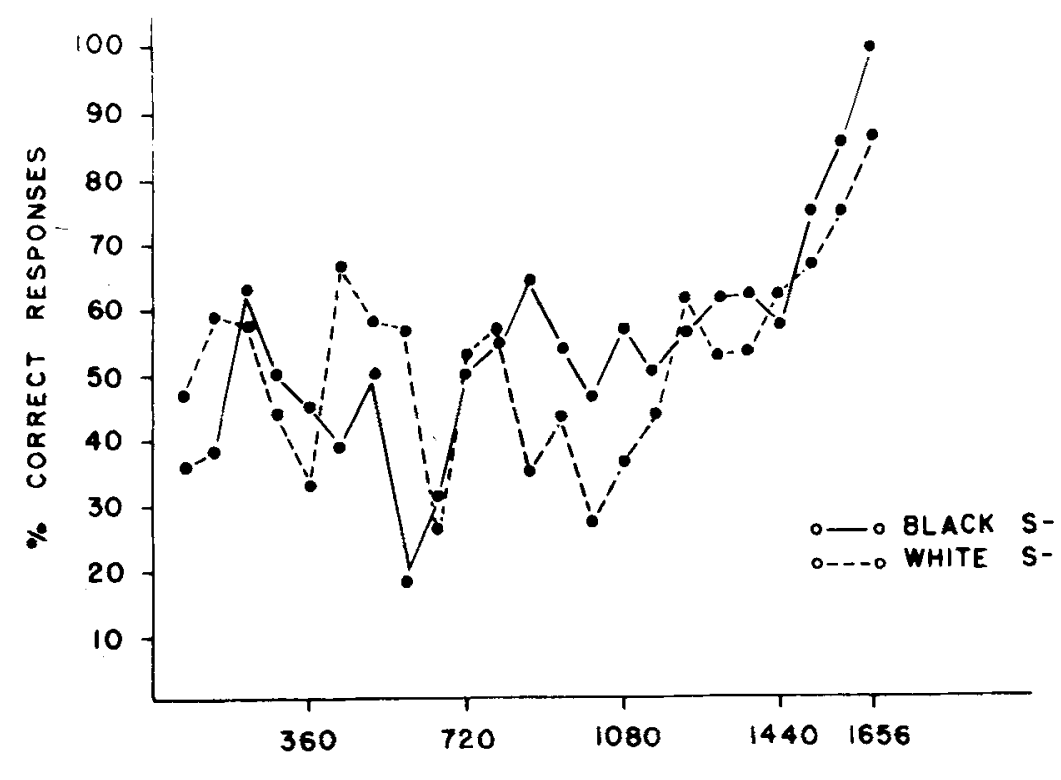

TRIALS

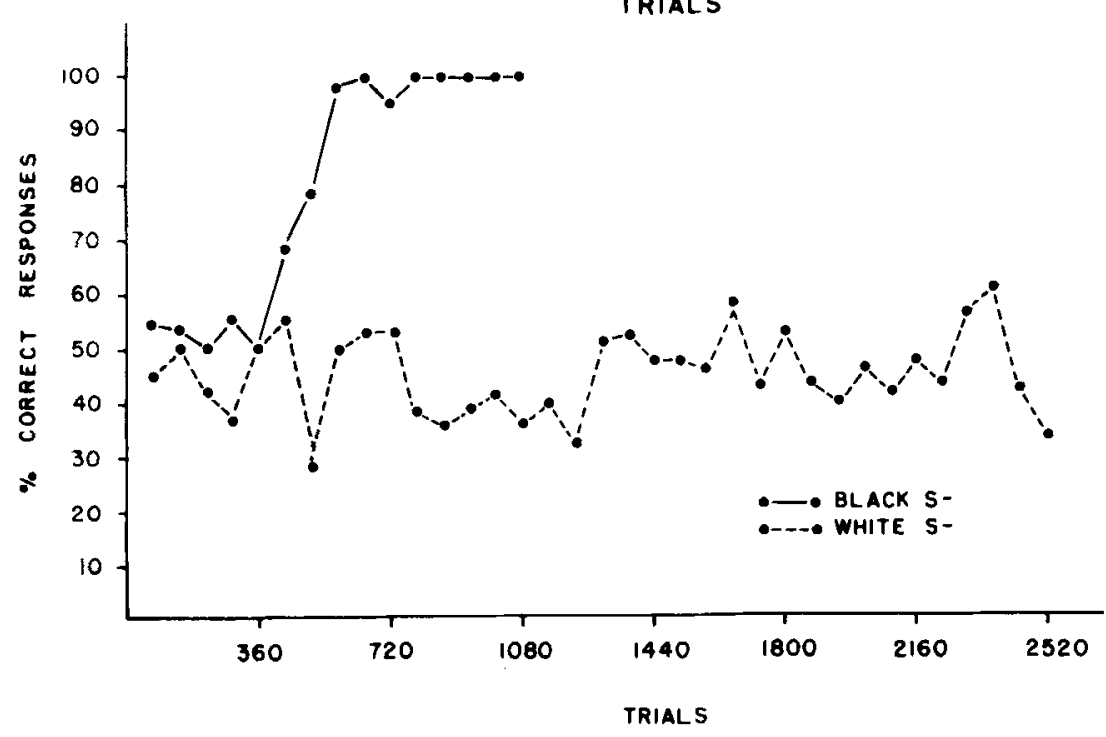

Fig. 1. (A) Skana's percent correct responses to each negative stimulus. Abscissa represents total presentations of both negative stimuli. (B) Hyak's percent correct responses to each negative stimulus. Abscissa represents total presentations of both negative stimuli.

for Hyak after 360 trials and remained in effect for 144 trials. Hyak had developed a position bias on trials presenting the white CS- after use of the black CS- was discontinued. A second correction period eliminated this preference. However, a bias was again developing when trial and error training ended.

Emotional behavior was frequent and most often developed during periods of incorrect responding. Such behavior was characterized by increased response latencies, stereotyped responding, and an increase in vocalizations following incorrect responses. Both Ss
CS+ and the white CS-. With errorless training, when only the CS+ was presented, 120 trials were required before Hyak responded consistently to this stimulus. During a further 60 trials, the white CS- was gradually reexposed with the $\mathrm{CS}+$ in four increments: the first 15 trials exposed $1 / 4$ of the CS-, the next 15 trials exposed $1 / 2$ of the CS-, and so on. At the end of the criterion block of 72 trials, errorless training totalled 252 trials.

The results of the study indicate that errorless training procedures have several advantages in training cetacea to make visual discriminations. While Hyak was hardly a naive $S$ at the introduction of errorless training, the number of trials to criterion using this procedure was small in comparison to the number of trials run without success under trial-and-error training. In addition, the procedure tends to eliminate incorrect responses and thereby reduces outbreaks of emotional behavior. With correction procedures introduced at the beginning of errorless training, the development of strong position preferences would be inhibited. Repeat trials, however, should not remain in effect indefinitely. Both killer whales developed a tendency to alternate the second response if the initial response was incorrect. During this period, responses on initial trials showed little improvement.

If more apparent stimulus parameters (e.g., brightness) are included to facilitate errorless training, these parameters themselves should be previously introduced using errorless training. In the study by Kellogg and Rice (1966), after displaying difficulties in solving the initial visual discrimination under trial and error training, the dolphin was trained on a brightness discrimination. The dolphin was then successfully transferred to the original visual discrimination problem using errorless training procedures. In general, it appears that elimination of nonrelevant stimulus parameters should, in the final stages, be made in reduced steps in order to transfer the cetacean successfully to the relevant discrimination with a minimum of errors and, hence, an adequate level of motivation.

\section{REFERENCES}

KELLOGG, W. N., \& RICE, C. E. Visual discrimination learning and problem solving in the bottlenose dolphin. In $\mathrm{K}$. $\mathrm{S}$. Norris (Ed.), Whales, dolphins and Norris (Ed.), Whales, dolphins and
porpoises. Los Angeles: University of California Press, 1966. Pp. 731-754.

SPONG, P., \& WHITE, D. Visual acuity and discrimination learning in the dolphin (Lagenorhynchus obliquidens). Experimental Neurology, 1971 , in press. 